\title{
Corela
}

Cognition, représentation, langage

$15-2 \mid 2017$

Vol. $15, n^{\circ} 2$

\section{Le désaccord travesti dans la comédie de mœurs de Molière : dialogisme et polyphonie}

Frédérique SAEZ

\section{OpenEdition}

\section{Journals}

Édition électronique

URL : http://journals.openedition.org/corela/5065

DOI : $10.4000 /$ corela.5065

ISSN : 1638-573X

Éditeur

Cercle linguistique du Centre et de I'Ouest - CerLICO

Référence électronique

Frédérique SAEZ, «Le désaccord travesti dans la comédie de mœurs de Molière : dialogisme et polyphonie », Corela [En ligne], 15-2 | 2017, mis en ligne le 05 décembre 2017, consulté le 19 avril 2019. URL : http://journals.openedition.org/corela/5065; DOI : 10.4000/corela.5065

Ce document a été généré automatiquement le 19 avril 2019

\section{cc)(1)(2)}

Corela - cognition, représentation, langage est mis à disposition selon les termes de la licence Creative Commons Attribution - Pas d'Utilisation Commerciale - Partage dans les Mêmes Conditions 4.0 International. 


\title{
Le désaccord travesti dans la comédie de mœurs de Molière : dialogisme et polyphonie
}

\author{
Frédérique SAEZ
}

\section{Liminaires}

\subsection{Objectifs}

1 Le présent article poursuit les recherches engagées lors du XXXIe colloque du CerLiCOㄹ. Nous avions alors examiné le phénomène de désaccord voilé dans deux de ses manifestations : le désaccord masqué et travesti.

2 Par désaccord voilé nous entendons le fait qu'un locuteur présente un point de vue non congruent par rapport à un élément antérieur ou postérieur ${ }^{2}$, mais exprime ce point de vue dissensuel de façon voilée - de fait ne l'exprime pas mais le laisse entendre. Nous avions alors également parlé de désaccord convenable, dans le sens où il permet de ménager les participants à l'échange, phénomène particulièrement représenté dans la comédie de mœurs du XVII siècle, d'où le choix de Molière.

3 Ce processus de désaccord voilé impose à l'interlocuteur - et à l'auditeur - une opération cognitive complexe de décodage, voire de co-énonciation dans le cas du désaccord travesti. Par conséquent nous avions opté pour une approche praxématique permettant de rendre compte à la fois des phénomènes cotextuels (linguistiques et/ou périverbaux) et des interactions verbales, donc de l'ensemble des mécanismes pragmatiques et acteurs à l'œuvre dans le désaccord voilé.

4 Nous nous intéresserons donc particulièrement aux phénomènes de dialogisme et/ou de polyphonie. En effet, le désaccord étant par essence ancré dans une antériorité plus ou moins immédiate et plus ou moins visible, on ne saurait postuler l'existence d'un discours dissensuel sans présupposer l'existence d'un discours (ou événement) antérieur auquel il s'oppose $^{3}$ : le désaccord est donc intrinsèquement dialogique au sens de Bakhtine (1984). 
5 Dans la mesure où le désaccord masqué a fait l'objet d'une précédente publication ${ }^{4}$ nous examinerons ici uniquement le désaccord travesti, en tentant de mettre en lumière les mécanismes de production et d'interprétation de ce désaccord par l'analyse des indices (linguistiques et/ou périverbaux) qui ouvrent l'interprétation d'un dissensus. Cette étude nous permettra d'aborder également la portée du désaccord.

6 En conclusion nous comparerons les deux types de désaccord voilé (masqué et travesti) du point de vue de la stratégie discursive mise en place par le locuteur et des différents rôles cognitifs des récepteurs.

\subsection{Intérêt du corpus de travail}

7 Le choix du drame comique classique révèle notre volonté de considérer le « périverbal intentionnel », soit la mimogestualité propre à un genre écrit (et chez Molière un écrit très contrôlé) pour être joué. Dans ce sens, les didascalies se présentent comme un outil précieux d'accès aux unités suprasegmentales (l'intonation, la mimogestualité etc.), sans avoir recours à notre intuition pour présumer d'un jeu de scène ou d'une émotion. On peut donc parler de périverbal intentionnel puisque les didascalies révèlent la volonté de l'auteur et jouent de fait pleinement leur rôle dans l'analyse du désaccord travesti.

Outre la mise en scène du non verbal, il appert également dans le genre dramatique une mise en abîme des actants - acteurs - de l'échange et de la construction du sens. En effet, en plus des personnages, il convient de considérer le public comme un allocutaire à part entière : il est le 'personnage' à qui s'adressent les indices du désaccord voilé car il en est l'instance de décodage (l'effet comique repose d'ailleurs sur l'interprétation d'une noncongruence entre les personnages). Il est le co-énonciateur immédiat et hors scène du discours scénique (linguistique et périverbal). Mais ce n'est pas la seule voix hors scène présente : il faut également considérer celle de l'auteur (Molière en l'occurrence) et celle de la «bienséance » (ou censure en d'autres termes).

Si donc le public peut être assimilé au ON-locuteur des théories de la polyphonie - comme vox populi - comment considérer les voix auteurs et censeurs qui hantent insidieusement le texte? Sont-ce ces voix qui justifient les masques du désaccord - voire parfois son travestissement?

10 En tout cas ces voix hors scène participent de la construction du sens, spécifiquement dans le cadre du désaccord travesti, qui n'est travesti que pour l'allocutaire scénique, mais non pour le public.

\subsection{Cadres théoriques et précisions terminologiques}

11 Sans prétendre trancher les questions terminologiques attachées aux domaines du dialogisme et des théories de la polyphonie, il est fondamental de clarifier la façon dont nous comprenons les termes employés ${ }^{6}$ dans la présente étude.

Nous prenons appui essentiellement sur les travaux de Bakhtine (1984), Nølke (1994, 2000 \& 2001), Fløttum (2001 \& 2002), et Anscombre (2009). Le cadre analytique est donc essentiellement celui de la théorie Scandinave de la Polyphonie en Linguistique (ScaPoLine), présentée comme "une théorie sémantique, discursive, structuraliste et instructionnelle. » (Nølke, 2001 : 1).

Deux choses entrent en jeu dans le phénomène de désaccord voilé : 
14 1. La plurivocité du sujet parlant: exprimant un point de vue dissensuel, il s'oppose nécessairement à un élément ${ }^{7}$ antérieur ou simultané dont son discours porte des traces. A priori donc, tout discours discordanciel est polyphonique ;

2. Le problème de la cohérence polyphonique ${ }^{8}$ (désormais c.p.) et du rôle du public. Selon Fløttum (2002: 338) «le récepteur s'attend à une cohérence polyphonique quand il aborde un texte [...]. [Il] s'attend à ce qu'il y ait une cohérence entre les différents points de vue exprimés. » Or ici nous examinerons des exemples dans lesquels cette cohérence semble mise en défaut. Par conséquent nous tenterons de comprendre comment est-ce que le récepteur la rétablit, l'élabore.

Pour Anscombre (2009), la polyphonie linguistique relève intrinsèquement de l' hétérogénéité énonciative dans le sens où " un énoncé est susceptible de faire entendre plusieurs voix, et non la seule voix de son locuteur/sujet parlant. » (ibid. : 13). Sans entrer dans les oppositions entre dialogisme et polyphonie, nous parlerons de polyphonie entendue dans le sens d'Anscombre (2009). Par conséquent, nous distinguerons entre deux cas de figures : 1 . des phénomènes dialogiques, où l'on observe la présence de plusieurs locuteurs qui correspondent chacun à un être au monde; et 2. polyphoniques, où un locuteur met en voix plusieurs points de vue par le biais d'énonciateurs.

18 Selon Olsen \& Nølke (2000: 54), au moins trois catégories de locuteurs (dits locuteurs virtuels) sont distinguables, selon l'emploi des pronoms :

19 3. Locuteur (LOC) : auquel renvoient les pronoms personnels de P1, il est l'auteur de l'énonciation ;

20 4. Allocutaire (ALL) : auquel renvoient les pronoms personnels de P2, il est le destinataire de l'énonciation;

21 5. Les troisièmes $\left(3^{\circ}\right)$ : auxquels renvoient les pronoms personnels de $\mathrm{P} 3$, ainsi que les syntagmes nominaux.

22 Il convient donc dans l'analyse de se situer à deux niveaux: celui de la production (encodage du désaccord voilé) et de la réception (interprétation discordancielle). Dans ces théories de la polyphonie ${ }^{9}$, LOC est considéré comme constructeur, i.e. comme l'instance discursive "responsable de l'énonciation et de l'énoncé qui en résulte.» (Fløttum, 2002 : 340). De plus,

24 LOC n'est pas une instance discursive univoque, mais plurivoque et l'on distingue au moins deux êtres discursifs internes au $\operatorname{LOC}^{10}$ :

25 6. Le locuteur de l'énoncé ( $\mathrm{L}$ ou $\mathrm{Ln}^{11}$ ) ou locuteur phrastique: image de LOC au moment de l'énonciation, donc multipliable autant qu'il y a d'énoncés ;

26 7. Le locuteur textuel (LT): image de LOC à un moment antérieur ou postérieur de l'énonciation. ${ }^{12}$

27 Le Ln met en scène des instances discursives nommées énonciateurs ${ }^{13}$, responsables des points de vue (pdv) contenus dans les énoncés. Selon Fløttum (2002 : 340, d'après Olsen \& Nølke 2000, et Nølke 2001), un pdv est une «unité qui renferme un contenu sémantique [et qui] a une source, appelée énonciateur. ». Interpréter un énoncé reviendrait donc à : 1 . identifier les différents pdv exprimés ; et 2. comprendre l'attitude énonciative de LOC par rapport au pdv exprimé par l'énonciateur. L'intérêt dans le cadre du désaccord voilé est que l'allocutaire-personnage n'interprète pas l'énoncé de la même façon que l'allocutaire-public, spécifiquement dans le désaccord 
travesti puisque l'allocutaire-personnage n'est pas censé accéder à l'interprétation dissensuelle, contrairement à l'allocutaire-public.

Anscombre (2009: 19) distingue trois types de liens possibles entre pdv et LOC qui peut: «a) s'identifier avec l'énonciateur, donc prendre en charge le pdv correspondant; b) se distancier ${ }^{14}$ de l'énonciateur, donc ne pas prendre en charge le pdv afférent; c) du seul fait de mettre en scène un énonciateur auquel on ne s'identifie pas et un pdv qu'on ne prend pas en charge, adopter un autre $\mathrm{pdv}$ [...]. ».

Le ON-locuteur (ON-Loc) est une entité crée par Anscombre (1990) d'après les travaux de Berrendonner (1981) et désigne une communauté linguistique dont le locuteur peut assumer la responsabilité. Par conséquent, les pdv exprimés par ON-Loc sont ON-vrai, i.e. admis par l'opinion générale, la vox populi ${ }^{15}$. Dans le genre dramatique, l'allocutaire-public peut être assimilé à ON-Loc et - de fait - prendre voix sur scène.

31 Si la ScaPoLine prévoie la possibilité aux allocutaires d'intervenir, pour Anscombre (2009: 16, note 19) toutefois il manque une étude générale sur ces êtres auditeurs, étude nécessaire dans la description du corpus considéré puisque le public ${ }^{16}$ semble ici participer à la construction du désaccord travesti.

Selon Olsen \& Nølke (2000: 55) il convient de poser, face au locuteur, un allocutaire (assimilé à ON-Loc dans les énoncés affirmatifs de la ScaPoLine) également plurivoque : 1 . l'allocutaire phrastique, qui est la personne présente dans la situation d'énonciation, ici l'auditeur-personnage (A\{personnage\}), et l'allocutaire textuel (AT), qui est une image passée ou future de cet auditeur-personnage, donc une projection de ce dernier. Il en va de même pour les troisièmes, divisibles en troisièmes phrastique (D) ou troisième textuel (DT) selon les mêmes critères que pour LOC et ALL.

De plus, si l'on reconnait à LOC la possibilité de se muer en ON-Loc, alors il faut postuler la présence d'un ON-Allocutaire (ON-All), qui correspondrait au public dans le genre dramatique. Ce ON-All équivaudrait à ce que Olsen \& Nølke (2000:64) nomment les interprétateurs, i.e. "les «spectateur» [qui] interprètent le drame selon [leurs] connaissances et attentes individuelles ». Ces "connaissances et attentes individuelles » peuvent être rapprochées de ce que la pragmasyntaxe fribourgeoise nomme la mémoire partagée ou mémoire discursive (désormais M), c'est-à-dire, selon Berrendonner (2002: 23), un « ensemble de représentations publiquement partagées [que l'on peut donc considérer comme] un monde virtuel commun [qui] subit en permanence des modifications. »

De fait, tous les partenaires de l'échange peuvent (se) référer à $M$ pour élaborer des inférences ou pour modifier un état de $\mathrm{M}$ : selon nous, ON-All participe à la construction du sens par recours à $\mathrm{M}$, accédant ainsi à l'interprétation discordancielle.

Les énonciateurs quant à eux, énoncent leurs pdv pour des destinataires et si LOC entretient un lien énonciatif avec les pdv, alors il convient de postuler également que ALL puisse entretenir un lien énonciatif vis-à-vis de l'attitude de LOC par rapport à ces pdv, et l'on peut postuler pour ALL les liens suivants :

a') coïncidence : le pdv de ALL coïncide avec l'attitude énonciative de LOC par rapport au $\mathrm{pdv}$;

b') non-coïncidence (ou distanciation) : le pdv de ALL ne coïncide pas avec l'attitude énonciative de LOC par rapport au pdv ;

c') élaboration par déduction inférentielle du pdv nouveau sous-entendu par LOC.

Le tableau ci-dessous met en regard les entités discursives et réceptives définies supra: 


\begin{tabular}{|c|c|c|c|c|}
\hline Niveau & \multicolumn{2}{|c|}{ Émission = sujet parlant } & \multicolumn{2}{|c|}{ Réception = auditeurs } \\
\hline \multirow{3}{*}{$\begin{array}{l}\text { Entités } \\
\text { discursives }\end{array}$} & \multirow{3}{*}{$\begin{array}{l}\text { LOC } \\
=\text { locuteur-comme- } \\
\text { constructeur } \\
=\text { responsable de } \\
\text { l'énonciation }\end{array}$} & $\begin{array}{l}\text { Ln } \\
=\text { personnage } \\
\text { scénique } \\
=\quad \text { locuteur } \\
\text { phrastique }\end{array}$ & \multirow{3}{*}{$\begin{array}{l}\text { ALL } \\
=\text { destinataire de } \\
\text { l'énonciation }\end{array}$} & $\begin{array}{l}\text { A\{personnage\} } \\
=\quad \text { personnage } \\
\text { scénique } \\
=\quad \text { allocutaire } \\
\text { phrastique }\end{array}$ \\
\hline & & $\begin{array}{l}\text { LT } \\
=\text { image de L } \\
=\quad \text { locuteur } \\
\text { textuel }\end{array}$ & & $\begin{array}{l}\text { AT } \\
=\text { image de A } \\
\text { \{personnage } \\
=\text { allocutaire textuel }\end{array}$ \\
\hline & & $\begin{array}{l}\text { ON-Loc } \\
=\text { vox populi }\end{array}$ & & $\begin{array}{l}\text { ON-Allocutaire } \\
=\text { public }\end{array}$ \\
\hline \multirow{3}{*}{$\begin{array}{l}\text { Liens } \\
\text { énonciatifs }\end{array}$} & \multicolumn{2}{|l|}{ a) responsabilité } & \multicolumn{2}{|c|}{ a') coïncidence à a) ou b) } \\
\hline & \multicolumn{2}{|l|}{ b) non-responsabilité } & \multicolumn{2}{|c|}{ b') non-coïncidence à a) ou b) } \\
\hline & \multicolumn{2}{|c|}{$\begin{array}{l}\text { c) non-responsabilité > ss-e pdv } \\
\text { nouveau }\end{array}$} & \multicolumn{2}{|c|}{ c') inférence du pdv nouveau ss-e. } \\
\hline
\end{tabular}

Nous avons conscience que les éléments cités dans cette section sont loin d'être évidents ni si simplistes qu'ils sont ici présentés. Mais comme ce n'est pas en soi l'objet de la présente communication, nous laisserons de côté les discussions afférentes, reconnaissant volontiers avoir ici simplifié les problématiques soulevées pour ne se concentrer que sur les besoins de notre description.

41 Voici un exemple de désaccord masqué qui permet d'illustrer les divers éléments :

8. $[R 1]^{17}$ DOM JUAN : Va, c'est une affaire entre le Ciel et moi, et nous la démêlerons bien ensemble, sans que tu t'en mettes en peine.

[R2] SGANARELLE : Ma foi Monsieur, j'ai toujours ouï dire que c'est une méchante raillerie que de se railler du Ciel, et que les libertins ne font jamais une bonne fin.

[R3] DOM JUAN : Holà maître sot, vous savez que je vous ai dit que je n'aime pas les faiseurs de remontrances.

[R4] SGANARELLE : Je ne parle pas aussi à vous, Dieu m'en garde. Vous savez ce que vous faites, vous; et si vous ne croyez rien, vous avez vos raisons; mais il y a de certains petits impertinents dans le monde, qui sont libertins sans savoir pourquoi, qui font les esprits forts, parce qu'ils croient que cela leur sied bien, et si j'avais un maitre comme cela, je lui dirais fort nettement, le regardant en face, osez-vous bien ainsi vous jouer du Ciel, et ne tremblez-vous point de vous moquer comme vous faites des choses les plus saintes [...] (ce n'est pas à vous que je parle, c'est à l'autre) pensez-vous dis-je que vous en soyez plus habile homme, que tout vous soit permis et qu'on n'ose vous dire vos vérités? apprenez de moi qui suis votre valet, que le Ciel punit tôt ou tard les impies, qu'une méchante vie amène une méchante mort et que...

(DJ, I,2)

En LOC et ALL sont respectivement Dom Juan, Sganarelle, et le public. En [R2] Sganarelle se cache dernière la vox populi (j'ai toujours oui dire) pour critiquer l'attitude de Dom Juan, 
et laisser entendre sa mort prochaine et violente (les libertins ne font jamais une bonne fin). Or [R3] (faiseur de remontrances) montre que Dom Juan accède à la discordance présente dans [R2] par le biais d'un discours doxatique rapporté, ce qui impose à Sganarelle un retrait immédiat : [R4] je ne parle aussi pas à vous.

Les indices anaphoriques ${ }^{18}$ ouvrent en outre dans $\mathrm{M}$ une dimension axiologique qui sera siège de la discordance déontique entre les personnages, et qui impose à Sganarelle la création immédiate [R4] d'un allocutaire fictif (je ne parle aussi pas à vous et ce n'est pas à vous que je parle, c'est à l'autre), marqué par LOC comme distinct de A\{Dom Juan\}, allocutaire fictif à qui s'adresse un locuteur textuel (je lui dirai fort nettement). Cette mise en abîme vocale va permettre à Sganarelle de critiquer ouvertement son maitre sans trop de risque.

En, ON-All infère un désaccord déontique des divers indices (masques) laissés par le locuteur (entres autres anaphores associatives et polyphonie interne, externe et (c)ouverte), et se présente donc de connivence avec LOC dans le sens où il est coïncident avec l'attitude énonciative de LOC.

Cet exemple montre la complexité des relations entre ces trois instances discursives, et signale la participation active de ON-All dans la construction de la cohérence polyphonique, et justifie la nécessité de prendre en compte tous les acteurs de la polyphonie.

\section{Le désaccord travesti}

Le désaccord travesti a tournure d'accord dans le sens où l'allocutaire-personnage ne saisit pas toujours le dissensus. Toutefois, si l'on se place du point de vue de l'auteur, alors il est nécessaire que ON-All infère ce dissensus, faute de rendre l'œuvre caduque et d'échapper à la visée comique.

La question sera donc de comprendre comment cette accession au dissensus est possible, ou dans quoi s'ancre - et par là même sur quoi porte - le désaccord.

Les exemples retenus sont regroupés selon les indices qu'ils comportent et qui ouvrent une possible interprétation dissensuelle.

\subsection{Ambigüités sémantico-référentielles}

49 Ces ambigüités peuvent être d'ordre sémantique (jeu sur la polysémie des termes) ou référentielle (jeux sur les pronoms endophoriques ou déictiques). C'est cette ambiguïté qui est exploitée par LOC, afin d'ouvrir une possible interprétation discordancielle, décodée par ON-All en référence à $\mathrm{M}$.

\subsubsection{Ambiguïtés sémantiques}

Soit des exemples comme :

9. [R1] SGANARELLE: Et n'y craignez-vous rien, Monsieur, de la mort de ce commandeur que vous tuâtes il y a six mois?

[R2] DOM JUAN : Et pourquoi craindre? Ne l'ai-je pas bien tué?

[R3] SGANARELLE : Fort bien, le mieux du monde, et il aurait tort de se plaindre.

$(D J, I, 2)$ 
10. [R1] ORGON : Le pauvre homme!

[R2] DORINE : Tous deux se portent bien enfin / Et je vais à Madame annoncer par

avance / La part que vous prenez à sa convalescence.

$(T, I, 4)$

51 En , [R3] joue sur la polysémie de tuer, entre manière (codes du duel respectés) et fait (le commandeur est vraiment mort), et montre - de façon travestie (fort bien) - le désaccord portant sur [R1] craindre (renforcé par la rime interne quasi paronymique craindre) plaindre) : ce que le valet déplore, c'est l'impiété de son maitre qui ne craint rien. Ici ONAll relève l'ambiguïté sémantique en raison de l'inadéquation de la réponse de Sganarelle à la question de Dom Juan fort bien : on ne peut pas être bien mort, on l'est ou pas, mais la mort se conçoit difficilement en terme de bien/mauvais dans la doxa. De fait, cette inadéquation pousse ON-All à chercher une cohérence ailleurs, et va construire le dissensus entre les personnages.

En , [R2] joue sur le sens de part qui désigne une fraction d'un tout divisé et le sens de la locution prendre part à qui signifie s'associer par la pensée, par le cœur à (une circonstance joyeuse ou douloureuse pour quelqu'un). Ici la construction syntaxique provoque l'ambiguïté qui serait levée si la part était postposé à prendre, or ici les deux lectures sont ouvertes ce que l'on peut ainsi reformuler :

11. Interprétation portion de : je vais à Madame annoncer la part que vous prenez à sa convalescence.

Interprétation empathie pour : je vais à Madame annoncer que vous prenez part à sa convalescence.

Le jeu sémantique permet donc à Orgon de sauver la face (la lecture prendre part à restant disponible), et à Dorine de suggérer une interprétation dissensuelle (elle n'est pas d'accord avec le comportement de son maitre) sans prendre de coup de bâton. Dorine d'ailleurs use d'autres éléments pour mettre encore plus de distance entre son maitre et sa critique, en effet, elle se transforme en locuteur représenté (LR) par le biais d'un discours rapporté (je vais annoncer à Madame) et transforme Orgon en allocutaire textuel ( vous).

De plus ici encore on note une inadéquation entre le sens du terme part qui implique au moins une portion d'un tout, même congrue, et la part nulle que prend Orgon dans la convalescence de sa femme ${ }^{19}$. C'est à nouveau de cette inadéquation que ON-All peut inférer le dissensus. En effet, à moins de considérer Dorine comme incohérente, et par là d'accepter l'inadéquation, la seule manière de rétablir une certaine cohérence est d'interpréter part comme de l'antiphrase, l'énoncé prenant alors une coloration ironique.

Dans ces deux exemples donc, on remarque que le locuteur a une voix doublée : il prend en charge un pdv (responsabilité) qu'il sous-entend injustifié car ce qu'il dit est inadéquat au regard de la situation. Cette double voix semble commentative, elle agit comme une sorte de parenthèse énonciative.

Qu'un LOC insère un pdv nouveau ss-e à partir d'un pdv dont il est responsable est un phénomène que nous avions observé dans le cadre du désaccord masqué ${ }^{20}$. Il semblerait que cette double voix, provenant d'un pdv pris en charge par LOC, puisse être rapprochée des êtres discursifs (désormais ê.d.) dits interpréteurs par Olsen \& Nølke (2000:62), i.e. «qui, par une interprétation (abstraite) de l'énoncé, reformule[nt] le pdv complexe (hérarchique et/ou relationnel) en pdv simple utilisable dans un pdv relationnel (ou hiérarchique ?) ». Pour les auteurs donc (ibid.: 63) «tout énoncé est susceptible de faire 
parler plusieurs voix, mais chaque voix, peut, à son tour contenir plusieurs voix discernables. »

De plus, dans la mesure où cet acte d'énonciation sous-jacent est anti-orienté par rapport au pdv immédiatement antérieur, on pourrait rapprocher ce phénomène polyphonique de la polyphonie interne entendue dans le sens de Fløttum (2002 : 353) d'après Nølke (1994), « dans le sens où ce sont les points de vue de L et de LT qui sont repérables. Il n'y a pas de pdv provenant d'êtres discursifs externes, c'est-à-dire différents du locuteur (LOC) avec ses deux images de L et de LT. "

Ici donc la polyphonie interne viendrait du dialogue entre Ln et Ln interpréteur (Lni).

Analyse dans le modèle :

\begin{tabular}{|c|c|c|c|}
\hline \multicolumn{2}{|c|}{ Émetteur } & \multicolumn{2}{|c|}{ Récepteur } \\
\hline \multicolumn{4}{|c|}{ Occurrence : ex. [R3] } \\
\hline L1 & $\begin{array}{l}\text { responsable pdv1 } \Delta^{21} \text { : fort bien, le mieux du monde et il aurait } \\
\text { tort de se plaindre }\end{array}$ & \multirow{3}{*}{$\begin{array}{l}\text { ON- } \\
\text { All }\end{array}$} & pdv1 inadéquat \\
\hline L1i & responsable pdv2 ss-e : pdv1 injustifié & & $\begin{array}{l}\text { rétablit c.p.: pdv1 } \\
=\text { antiphrase }\end{array}$ \\
\hline & & & $\begin{array}{l}\text { construit } \\
\text { discordance } \\
\text { LOC vs ALL }\end{array}$ \\
\hline \multicolumn{4}{|c|}{ Occurrence : ex. [R2] } \\
\hline L1 & responsable pdv1: je vais annoncer à Madame & \multirow{4}{*}{$\begin{array}{l}\text { ON- } \\
\text { All }\end{array}$} & $\begin{array}{l}\text { transforme L1 en } \\
\text { LR }\end{array}$ \\
\hline LR & $\begin{array}{l}\text { responsable de pdv2: la part que vous prenez à sa } \\
\text { convalescence }\end{array}$ & & pdv2 inadéquat \\
\hline$L R i$ & responsable pdv3 ss-e : pdv2 injustifié & & $\begin{array}{l}\text { rétablit c.p.: pdv2 } \\
=\text { antiphrase }\end{array}$ \\
\hline & & & $\begin{array}{l}\text { construit } \\
\text { discordance } \\
\text { LOC vs ALL }\end{array}$ \\
\hline
\end{tabular}

Il semble que ce phénomène de polyphonie interne spécifique, où LOC s'oppose à luimême comme interpréteur, puisse être rapproché de ce que l'on peut observer lors d'une ambiguité référentielle.

\subsubsection{Ambiguités référentielles}

61 Soit : 
12. [R1] DOM JUAN : Et ne trouves-tu pas que j'ai raison d'en user de la sorte?

[R2] SGANARELLE : Eh Monsieur.

[R3] DOM JUAN : Quoi ? Parle.

[R4] SGANARELLE : Assurément que vous avez raison, si vous le $\mathbf{e}_{1}$ voulez. On ne peut pas aller là contre; mais si vous ne $\mathbf{l e}_{2}$ vouliez pas, ce serait peut-être une autre affaire.

(DJ, I,2) univers fictionnel (confirmé par le conditionnel subséquent (serait) doublé de l'adverbe peut-être) dans lequel Sganarelle peut asséner à un allocutaire fictif ${ }^{24}$ (AF), représenté par vous et coréférent à l'AT, ce qui serait possible à condition que cet $\mathrm{AF}$ modifie son comportement, ce que l'on peut ainsi formuler :

14. [R4] mais si vous ne le vouliez pas, ce serait peut-être une autre affaire.

Paraphrase 3 : si vous ne vouliez pas en user de la sorte ce serait peut-être une autre affaire

Paraphrase 4 : si vous ne vouliez pas avoir raison ce serait peut-être une autre affaire

Analyse dans le modèle :

\begin{tabular}{|l|l|l|l|}
\hline \multicolumn{2}{|l|}{ Emetteur } & \multicolumn{2}{l|}{ Récepteur } \\
\hline \multirow{2}{*}{ L1 } & $\begin{array}{l}\text { responsable pdv1 : assurément que vous } \\
\text { avez raison }\end{array}$ & $\begin{array}{l}\text { ON- } \\
\text { All }\end{array}$ & $\begin{array}{l}\text { transforme A\{Dom Juan\} en AT : } \\
\text { vous' } \\
\text { ouvre ambiguïté référentielle }\end{array}$ \\
\cline { 2 - 3 } & responsable pdv2: si vous le voulez &
\end{tabular}




\begin{tabular}{|c|c|c|}
\hline L1i & responsable pdv3 ss-e : pdv1 injustifié & rétablit c.p. : pdv1 = antiphrase \\
\hline \multirow{2}{*}{$\begin{array}{l}\text { ON- } \\
\text { Loc }\end{array}$} & $\begin{array}{l}\text { non-responsable pdv4: on peut aller là } \\
\text { contre }\end{array}$ & \multirow{2}{*}{ introduit ON-Loc } \\
\hline & responsable pdv5 ss-e : pdv4 injustifié & \\
\hline \multirow{4}{*}{ L2 } & responsable pdv6 : pdv5 injustifié mais & $\begin{array}{l}\text { introduit L2: 'mais' } \\
\text { pose contre-argumentation: } \\
\text { L2 vs ON-Loc }\end{array}$ \\
\hline & non-responsable pdv7 : si vous le vouliez & \multirow{3}{*}{$\begin{array}{l}\text { transforme AT en AF tel que AF = } \\
\neg \mathrm{A}\{\mathrm{DJ}\} \\
\text { confirme ambiguïté référentielle }\end{array}$} \\
\hline & responsable pdv8 ss-e : pdv7 injustifié & \\
\hline & $\begin{array}{l}\text { responsable pdv9: ce serait peut-être une } \\
\text { autre affaire. }\end{array}$ & \\
\hline & & construit discordance : LOC vs ALL \\
\hline
\end{tabular}

72 Ici le recours à la polyphonie permet à Sganarelle de travestir son désaccord de sorte qu'il ne soit pas interprété comme tel par son maitre. Dans cette réplique, un locuteur (L1) prend en charge de se montrer d'accord avec l'allocutaire-personnage, accord immédiatement réfuté un ê.d. interpréteur (L1i), nuancé par ON-Loc, et contre-argumenté par L2 qui par l'usage d'une construction hypothétique (si + imparfait-conditionnel), permet la création d'un avatar de ALL (soit AF) à qui l'on peut laisser entendre, d'un point de vue argumentatif, qu'il a tort.

73 En effet, la suite possible des deux paraphrases retenues en ( montre la double portée de ce désaccord travesti et donc son haut rendement dans la stratégie argumentative de Sganarelle :

15. [R4] mais si vous ne le vouliez pas, ce serait peut-être une autre affaire.

Paraphrase 3': si vous ne le (/en user de la sorte) vouliez pas, ce serait peut-être une autre affaire, et vous pourriez renoncer à une vie de libertin.

Paraphrase 4' : si vous ne le (/avoir raison) vouliez pas, ce serait peut-être une autre affaire et il serait possible de vous faire entendre raison.

74 La paraphrase 3' porte sur le comportement de Dom Juan, lorsque la paraphrase 4' porte sur son caractère. Travestir le désaccord lui offre ici une double portée. De plus il semble que ce soit justement la polyphonie qui permette ici à ON-All d'interpréter le dissensus.

\subsection{Polyphonie interne}

75 Les exemples infra reposent sur un phénomène de polyphonie interne analogue à et qui est confirmé par la présence du connecteur mais ou le double jeu du comédien. Dans tous les cas, LOC se révèle en contradiction avec lui-même.

\subsubsection{Connecteur contre-argumentatif mais}

Le connecteur mais signale que le locuteur ne prend pas la responsabilité de ce qui a été énoncé par le locuteur antérieur, ainsi dans : 
16. [R1] SGANARELLE : Mais, Monsieur, cela serait-il de la permission que vous m'avez donnée, si je vous disais que je suis tant soit peu scandalisé de la vie que vous menez?

[R2] DOM JUAN : Comment? Quelle vie est-ce que je mène?

[R3] SGANARELLE : Fort bonne. Mais, par exemple, de vous voir tous les mois vous marier comme vous faites..

$(D J, I, 2)$

17. [R1] ORONTE: Est-ce que vous voulez me déclarer, par-là, / Que j'ai tort de vouloir...

[R2] ALCESTE : Je ne dis pas cela ${ }^{25}$ : / Mais je lui disais, moi, qu'un froid Ecrit assomme, $[. .$.

$(M, I, 2)$

Ici les assertions positive fort bonne ou négative je ne dis pas cela, sont saisies par ON-All comme des antiphrases en raison de ce qu'elles sont inadéquates par rapport à la situation. Cette interprétation est confirmée par présence de mais, ce que l'on pourrait ainsi paraphraser : fort mauvaise et je dis bien cela.

Cette antiphrase est prise en charge par le locuteur interpréteur, d'où une première polyphonie interne. ON-All décode cette antiphrase par référence dans $M$ à ses connaissances de l'œuvre et des rapports entre les personnages. De fait, s'il prend les énoncés fort bonne et je ne dis pas cela de façon littérale, il y a incohérence entre l'énoncé et la situation : la vie menée par Dom Juan n'est pas bonne du point de vue de la morale, et les vers de Oronte sont manifestement mauvais, il ferait mieux d'arrêter d'écrire. Par conséquent ON-All rétablit la cohérence polyphonique en comprenant les mentions fort bonne et je ne dis pas cela comme antiphrastiques, et construit la discordance entre les personnages.

Mais cette forme de polyphonie interne par l'interpréteur n'est pas la seule et en effet, la présence du connecteur impose à ALL l'introduction dans M d'un second LOC, antiorienté par rapport au premier, et donc co-orienté par rapport à l'interpréteur. Ce lien énonciatif entre le second locuteur et le pdv antérieurement exprimé est appelé contreargument par Fløttum (2002: 348).

Analyse dans le modèle :

\begin{tabular}{|c|c|c|c|}
\hline \multicolumn{2}{|c|}{ Émetteur } & \multicolumn{2}{|c|}{ Récepteur } \\
\hline \multicolumn{4}{|c|}{ Occurrence : ex. [R5] } \\
\hline L1 & responsable pdv 1 : fort bonne & \multirow{5}{*}{$\begin{array}{l}\text { ON- } \\
\text { All }\end{array}$} & pdv1 inadéquat \\
\hline L1i & $\begin{array}{l}\text { responsable pdv2 ss-e : pdv } 1 \\
\text { injustifié }\end{array}$ & & rétablit c.p. : pdv1 = antiphrase \\
\hline \multirow[t]{2}{*}{ L2 } & contre-argument pdv1: mais & & $\begin{array}{l}\text { introduit L2 ('mais') tel que : } \\
\text { L2 et L1 : anti-orientés ; } \\
\text { L2 et L1i : co-orientés }\end{array}$ \\
\hline & $\begin{array}{l}\text { responsable pdv3: de vous } \\
\text { voir }[\ldots]\end{array}$ & & transforme A\{Dom Juan $\}$ en AT : 'vous' \\
\hline & & & construit discordance : LOC vs ALL \\
\hline
\end{tabular}




\begin{tabular}{|c|c|c|c|}
\hline \multicolumn{4}{|c|}{ Occurrence : ex. [R2] } \\
\hline \multirow{2}{*}{ L1 } & $\begin{array}{l}\text { non-responsable pdv1: je dis } \\
\text { cela }\end{array}$ & \multirow{6}{*}{$\begin{array}{l}\text { ON- } \\
\text { All }\end{array}$} & \multirow{2}{*}{ pdv2 inadéquat } \\
\hline & $\begin{array}{l}\text { responsable pdv2 ss-e : pdv } 1 \\
\text { injustifié }\end{array}$ & & \\
\hline$L 1_{i}$ & $\begin{array}{l}\text { responsable pdv3 ss-e : pdv1 } \\
\text { justifié }\end{array}$ & & rétablit c.p. : pdv2 = antiphrase \\
\hline \multirow[t]{2}{*}{ L2 } & $\begin{array}{l}\text { contre-argument pdv2 ss-e: } \\
\text { mais }\end{array}$ & & $\begin{array}{l}\text { introduit L2 ('mais') tel que : } \\
\text { L2 et L1 : anti-orientés ; } \\
\text { L2 et } L 1_{i} \text { co-orientés. }\end{array}$ \\
\hline & $\begin{array}{l}\text { responsable pdv } 3: \text { je lui } \\
\text { disais }[. . .]\end{array}$ & & $\begin{array}{l}\text { transforme DT ('lui') en AF \& L2 en LR ('disais'), tel } \\
\text { que : } \mathrm{AF}=\mathrm{A}\{\text { Oronte }\}\end{array}$ \\
\hline & & & construit discordance : LOC vs ALL \\
\hline
\end{tabular}

81 La double polyphonie ( $\mathrm{Ln}-\mathrm{Ln}_{i} \&$ L1-L2) ouvre sur un jeu complexe d'argument/contreargument (connecteur mais) et présente l'avantage insigne de permettre à l'allocutairepersonnage de sauver la face, voire, de ne pas accéder au dissensus, contrairement à l'allocutaire-public qui peut - de fait - l'interpréter. Par conséquent l'association $\left[\operatorname{Ln}_{i}+\right.$ connecteur contre-argumentatif mais] se présente comme un élément privilégié dans la construction du désaccord travesti.

\subsubsection{Modalité interrogative ( $\&$ jeu de scène)}

Dans ces exemples, les didascalies montrent un jeu frôlant l'indécence pour et l'inconvenance pour :

18. [R1] TARTUFFE : c'est par excès de zèle. / De vous faire aucun mal je n'eus jamais dessein, / Et j'aurais bien plutôt...

Il lui met la main sur le genou

[R2] ELMIRE : Que fait là votre main?

( $T$, III,3)

19. [R1] ORONTE : [...] Je crois qu'un Ami chaud, et de ma Qualité, / N'est pas, assurément, pour être rejeté. /C'est à vous, s'il vous plait, que ce discours d'adresse. En cet endroit Alceste parait tout rêveur et semble n'entendre pas qu'Oronte lui parle.

[R2] ALCESTE : A moi, Monsieur?

$(M, I, 2)$

En , Elmire souligne par l'interrogation sa désapprobation, modalité qui permet de ménager l'honneur de Tartuffe. Il en va de même en dans lequel Alceste feint de n'avoir pas entendu Oronte s'adresser à lui. Les locuteurs se servent donc d'une modalité interrogative pour exprimer leur désaccord par rapport à la situation dans laquelle ils se trouvent et qui est provoquée par la présence d'un autre personnage, ce que l'on pourrait ainsi interpréter :

${ }_{[\mathrm{R} 2]}$ : votre main n'a rien à faire sur mon genou, retirez-la.

$[\mathrm{R} 2]$ : je n'ai aucune envie de vous écouter, retirez-vous. 
All ne peut construire ces inférences que par référence dans $M$ à ses connaissances de l'œuvre et des rapports entre les personnages. La modalité interrogative apparait dès lors inadéquate par rapport à la situation (Tartuffe mériterait un soufflet et Oronte est un importun), à moins d'être réinterprétée ainsi qu'en par Ln interpréteur. Par conséquent, l'interprétation dissensuelle apparait ici garante de la cohérence polyphonique puisqu'elle résout l'inadéquation entre la modalité interrogative et la situation qui aurait appelé un impératif.

Analyse dans le modèle :

\begin{tabular}{|c|c|c|c|}
\hline Em & etteur & \multicolumn{2}{|c|}{ Récepteur } \\
\hline \multicolumn{4}{|c|}{ Occurrence : ex. \& [R2] } \\
\hline L1 & $\begin{array}{l}\text { responsable pdv1: } \\
\text { : que fait là votre main? } \\
\text { : à moi Monsieur? }\end{array}$ & \multirow{3}{*}{ ON-All } & pdv1 inadéquat \\
\hline$L 1_{i}$ & $\begin{array}{l}\text { responsable pdv2 ss-e : } \\
\text { : retirez votre main! } \\
\text { : ne me parlez pas! }\end{array}$ & & rétablit c.p. : coïncident $L 1 i$ \\
\hline & & & construit discordance : LOC vs ALL \\
\hline
\end{tabular}

\subsection{Polyphonie ouverte ( ?) : cas particuliers}

Olsen \& Nølke (2000 : 90 et suiv.) parlent de polyphonie ouverte dans le cadre du discours indirect libre. Dans ce cadre les auteurs introduisent un nouvel être discursif, le locuteur représenté (LR) qui est l'Autre dont on rapporte les propos. Or le discours de LR peut être intégré de deux manières dans le discours de LOC, en effet, ce dernier peut (ibid.: 89) « DIRE ${ }^{26}$ l'acte d'énonciation de LR en le (re)présentant au moyen d'un inquit (c'est le discours rapporté [...]) [ou] MONTRER l'acte d'énonciation de LR en le (re)présentant sans inquit (c'est le discours libre)».

Cela implique que si LOC choisit de dire l'acte d'énonciation de LR, alors s'agit de polyphonie ouverte dans laquelle les deux voix de LOC et LR peuvent être prises à part ; mais si LOC choisit de montrer l'acte d'énonciation de LR, il s'agit de polyphonie couverte (v. section infra) et alors les voix de LOC et LR ne peuvent être séparées et LOC est si bien caché qu'il disparait parfois complètement (ibid. : 91).

\subsubsection{Double jeu : didascalies}

Ici le jeu de scène explicité par l'auteur vient confirmer la dualité des personnages. Il ne s'agit donc pas d'une polyphonie ouverte au sens stricte, mais d'une forme particulière dans laquelle ${ }^{27}$ on pourrait considérer que les didascalies - qui explicitent le jeu des personnages et le double discours qu'ils tiennent - fonctionnent comme des inquit. De fait, on observe la création d'un LR particulier également car anti-orienté par rapport à LOC et entièrement fictif ${ }^{28}\left(\mathrm{LR}_{\mathrm{F}}\right)$. 
89 Toutefois, dans la mesure où LOC emploi 'je' indifféremment pour LR et lui-même, on peut considérer ce LR comme ayant un discours dissociable de celui de LOC, donc un cas particulier de polyphonie ouverte.

Soit les exemples suivants :

20. [R1] BELINE, feignant de pleurer: Ne me parlez point de cela, je vous prie, vous me faites mourir de frayeur... Elle se ravise et lui dit : Combien dites-vous qu'il y a d'argent comptant dans votre alcôve?

[R2] ARGAN : Vingt mille francs, mon Cœur.

[R3] BELINE : Tous les biens de ce monde ne me sont rien en comparaison de vous... De combien sont les deux billets?

(MI, I,7)

21. TOINETTE, par dérision: Ma foi, Monsieur, je suis pour vous maintenant, et je me dédis de tout ce que je disais hier. Voici Monsieur Diafoirus le Père, et Monsieur Diafoirus le Fils qui viennent vous rendre visite. Que vous serez bien engendré ! Vous allez voir le garçon le mieux fait du monde et le plus spirituel. Il n'a dit que deux mots qui m'ont ravie, et votre fille va être charmée de lui. (MI, II,4)

91 En , les didascalies révèlent la duplicité du personnage, et en la didascalie montre un revirement factice.

En effet, pour ON-All, c'est l'inadéquation entre le «dit» du personnage et notre connaissance de la pièce qui permet de comprendre, d'inférer, la contre-argumentation entre le jeu et le dit. Si en Beline double son jeu d'un double langage, en LOC reste linguistiquement caché. De plus, ON-All saisit le 'vrai' dans la dualité des personnages, identifiant les propos de $\mathrm{LR}_{\mathrm{F}}$ comme 'faux' en regard de ses connaissances de l'œuvre et $\mathrm{du}$ jeu d'acteur. Les didascalies - ou le jeu de scène - viennent donc confirmer cette inadéquation entre le dit et la situation et donc la cohésion polyphonique est assurée par la construction d'un désaccord entre A\{personnage\} et LOC.

Analyse dans le cadre :

\begin{tabular}{|c|c|c|c|}
\hline \multicolumn{2}{|c|}{ Emetteur } & \multicolumn{2}{|c|}{ Récepteur } \\
\hline \multicolumn{4}{|c|}{ Occurrences : ex. [R1] + [R3] } \\
\hline \multirow[t]{2}{*}{$\mathrm{LR}_{\mathrm{F}}$} & $\begin{array}{l}\text { non-responsable pdv } 1 \text { : } \\
-[\mathrm{R} 1] \text { parlez-moi de cela } \\
-[\mathrm{R} 3] \text { tous les biens }[. . .] \text { quelque chose }\end{array}$ & \multirow{4}{*}{$\begin{array}{l}\text { ON- } \\
\text { All }\end{array}$} & \multirow[t]{2}{*}{$\begin{array}{l}\mathrm{M}_{\text {\{épistémè\} }}: \mathrm{pdv} 2 \text { ss-e inadéquat } \\
\text { rétablit c.p. : transforme LOC en } \mathrm{LR}_{\mathrm{F}}\end{array}$} \\
\hline & responsable de pdv2 ss-e : pdv 1 injustifié & & \\
\hline \multirow[t]{2}{*}{ L1 } & $\begin{array}{l}\text { responsable pdv3 : } \\
-[\mathrm{R} 1] \text { Combien dites-vous }[\ldots] \\
-[\mathrm{R} 3] \text { de combien sont les deux billets? }\end{array}$ & & $\begin{array}{l}\text { introduit par } \mathrm{M}_{\{\text {jeu acteur\} }} \mathrm{L} 1 \text { tel que } \mathrm{L} 1 \\
v s \mathrm{LR}_{\mathrm{F}} \\
\text { interprète } \mathrm{LR}_{\mathrm{F}} \text {-Faux vs } \mathrm{L} 1 \text {-Vrai }\end{array}$ \\
\hline & & & construit discordance : L1 vs ALL \\
\hline \multicolumn{4}{|c|}{ Occurrence : ex. } \\
\hline & responsable pdv1 : je suis [...] maintenant & \multirow{2}{*}{$\begin{array}{l}\text { ON- } \\
\text { All }\end{array}$} & \\
\hline $\mathrm{LR}_{\mathrm{F}}$ & responsable pdv2 : je me dédis [...] hier & & $\mathrm{M}_{\{\text {\{épistémè\} }}: \mathrm{pdv1,2} \& 3$ inadéquats \\
\hline
\end{tabular}




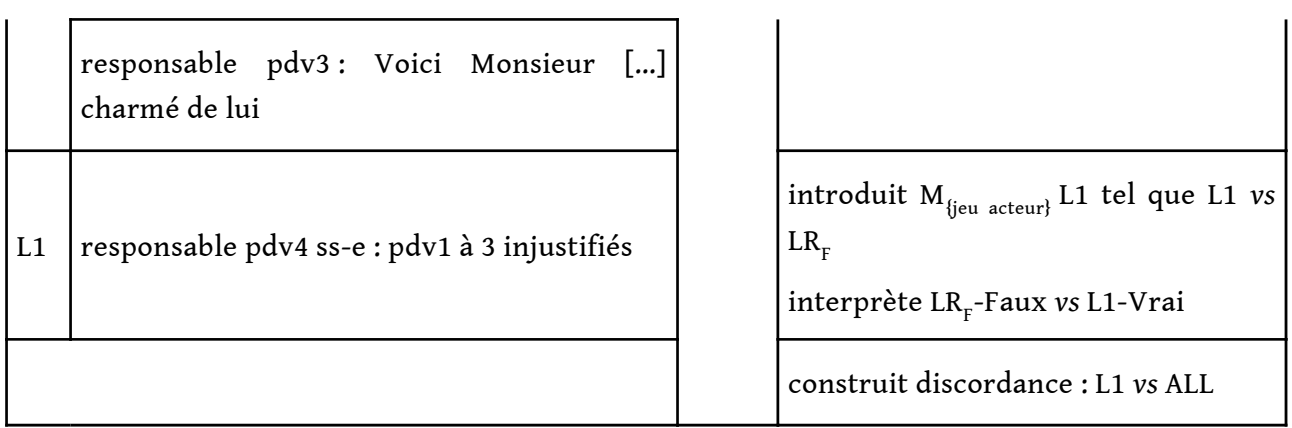

L'analyse dans le cadre permet de montrer que la nécessité d'interpréter et co-construire l'énonciation contraint ON-All à prendre position dans un jeu où le LOC se contredit luimême, dans le sens où il interprète - guidé par la didascalie - $\mathrm{LR}_{\mathrm{F}}$-faux. Cette interprétation se fait nécessairement par référence dans $M$ à ses connaissances de l'œuvre ou grâce à l'observation du jeu d'acteur. Ici donc ON-All n'est plus un simple spectateur mais un véritable spectacteur ${ }^{29}$, à la fois récepteur et co-énonciateur du texte. Sans ce travail de co-énonciation de ON-All, la cohésion polyphonique ne peut être assurée.

\subsubsection{Double langage et langage double (aparté) : un locuteur en contradiction}

Dans ces exemples il s'agit, un peu sur le modèle des précédents, de cas où le locuteur se contredit lui-même. Ici encore, la notion de polyphonie ouverte est à adapter car on peut considérer que ce ne sont plus les didascalies qui signalent un double langage, fonctionnant de fait comme un inquit, mais LOC lui-même, en se contredisant seul.

LOC agit donc à l'envers de la métarègle de non-contradiction établie par Charolles (1978 : 22). Or c'est cette entorse qui fonctionne justement comme le signale d'un double jeu et force, ici aussi, ON-All à introduire dans $\mathrm{M}$ la présence d'un $\mathrm{LR}_{\mathrm{F}}$ qu'un $\mathrm{Ln}$ va venir contredire dans l'aparté. Ln est donc lui-même doublé d'un interpréteur qui vient asséner - comme une sorte de parenthèse discursive - que le discours tenu par $\mathrm{LR}_{\mathrm{F}}$ est faux, car Ln n'en est pas responsable.

ON-All est donc ici encore un co-énonciateur.

Soit le corpus suivant :

22. [R1] DOM JUAN : Allons vite, c'est trop d'honneur que je vous fais, et bien heureux est le valet qui peut avoir la gloire de mourir pour son maitre.

[R2] SGANARElle: Je vous remercie d'un tel honneur. ô Ciel, puisqu'il s'agit de mort, fais-moi la grâce de n'être point pris pour un autre.

$(\mathrm{DJ}, \mathrm{II}, 5)$

23. [R1] PHILAMINTE : Comment? Vous avez peur d'offenser la Coquine? / Vous lui parlez d'un ton tout à fait obligeant?

[R2] CHRISALE bas : Moi ? Point. Allons sortez. Va-t-en, ma pauvre Enfant.

$(F s, \mathrm{II}, 6)$

24. [R1] DOM JUAN : Eh mourez, le plus tôt que vous pourrez, c'est le mieux que vous puissiez faire ; il faut que chacun vive son tour, et j'enrage de voir que des Pères vivent autant que leurs fils.

[R2] SGANARELLE : Ah Monsieur, vous avez tort.

[R3] DOM JUAN : J'ai tort ?

[R4] SGANARELLE : Monsieur...

[R5] DOM JUAN : J'ai tort? 
[R6] SGANARELLE : Oui Monsieur vous avez tort, d'avoir souffert ce qu'il vous a dit, et vous le deviez mettre dehors par les épaules; a-t-on jamais vu rien de plus impertinent ? un père venir faire des remontrances à son fils, et lui dire de corriger ses actions, de se ressouvenir de sa naissance, de mener une vie d'honnête homme, et cent autres sottises de pareille nature; cela se peut-il souffrir à un homme comme vous qui savez comme il faut vivre ?j'admire votre patience, et si j'avais été en votre place, je l'aurais envoyé promener. ô complaisance maudite ! à quoi me réduis-tu?

$(D J, I V, 5)$

\begin{tabular}{|c|c|c|c|}
\hline Eme & tteur & \multicolumn{2}{|c|}{ Récepteur } \\
\hline \multicolumn{4}{|c|}{ Occurrence : ex. [R2] / / [R6] } \\
\hline LRF & $\begin{array}{l}\text { responsable pdv1: } \\
\text { - je vous remercie d'un tel honneur. } \\
\text { - Allons sortez! } \\
\text { - }{ }^{30} \text { Oui Monsieur [...] promener. }\end{array}$ & \multirow{4}{*}{ ON-All } & $\begin{array}{l}\mathrm{M}_{\text {\{épistémè\} }}: \text { pdv1 inadéquat } \\
\text { rétablit c.p. : transforme LOC en } \mathrm{LR}_{\mathrm{F}}\end{array}$ \\
\hline \multirow[t]{2}{*}{ L1 } & $\begin{array}{l}\text { responsable pdv2: } \\
\text { - ô Ciel, [...] pris pour un autre. } \\
\text { - Va-t-en, ma pauvre Enfant! } \\
\text { - Ô complaisance [...] réduis-tu? }\end{array}$ & & \multirow[t]{2}{*}{$\begin{array}{l}\text { introduit } \mathrm{L} 1 \text { tel que } \mathrm{L} 1 \text { vs } \mathrm{LR}_{\mathrm{F}} \\
\text { interprète } \mathrm{LR}_{\mathrm{F}} \text {-Faux vs } \mathrm{L} 1-\text {-Vrai }\end{array}$} \\
\hline & responsable pdv3 ss-e : pdv1 injustifié & & \\
\hline & & & construit discordance : L1 vs ALL \\
\hline
\end{tabular}

Ici l'aparté fonctionnerait comme un inquit nous permettant d'interpréter que le discours de L1 est un contre-argument à celui de $\mathrm{LR}_{\mathrm{F}}$. Dans ce sens il s'agirait bien d'un tour particulier de polyphonie ouverte, car les actes d'énonciation de $\mathrm{LR}_{\mathrm{F}}$ et de $\mathrm{L} 1$ sont clairement dissociables. 


\subsection{Polyphonie couverte}

Dans les exemples infra rien ne vient confirmer le double jeu des personnages, de fait, c'est uniquement l'inadéquation entre la situation et le discours qui permet à ON-All d'inférer depuis $M$ un désaccord, auquel n'accède pas l'allocutaire-personnage. En effet, à moins de considérer l'émetteur comme fou, les exemples qui suivent seraient considérés comme ineptes si l'on n'inférait pas un cas de polyphonie couverte où LOC montre un pdv avec lequel il est en désaccord.

Soit les exemples suivants :

25. [R1] DOM JUAN : [...] et j'ai une petite barque avec des gens avec quoi fort facilement je prétends enlever la belle.

[R2] SGANARELLE : Ha, Monsieur!

[R3] DOM JUAN : Hein?

[R4] SGANARELLE : C'est fort bien fait à vous, et vous le prenez comme il faut. Il n'est rien en ce monde que de se contenter.

(DJ, I,2)

26. [R1] VALÈRE : Sans dot?

[R2] HARPAGON : Oui.

[R3] VALÈRE: Ah, je ne dis plus rien. Voyez-vous, voilà une raison tout à fait convaincante ; il faut se rendre à cela. [...]

[R4] HARPAGON : Sans dot.

[R5] VALÈRE : Il est vrai. Cela ferme la bouche à tout, sans dot. Le moyen de résister à une raison comme celle-là?

(L'A, I,5)

27. [R1] MONSIEUR DIAFOIRUS : A vous en parler franchement, notre métier auprès des Grands ne m'a jamais paru agréable, et j'ai toujours trouvé qu'il valait mieux pour nous autres, demeurer au public. Le public est commode : vous n'avez à répondre de vos actions à personne, et pourvu que l'on suive le courant des règles de l'Art, on ne se met point en peine de tout ce qui peut arriver. Mais ce qu'il y a de fâcheux auprès des grands, c'est que quand ils viennent à être malades, ils veulent absolument que leurs Médecins les soignent.

[R2] TOINETTE : Cela est plaisant, et ils sont bien impertinents de vouloir que vous autres Messieurs vous les guérissiez. Vous n'êtres point auprès d'eux pour cela. Vous n'y êtes que pour recevoir vos pensions et leur ordonner les Remèdes, c'est à eux à guérir s'ils peuvent.

[R3] MONSIEUR DIAFOIRUS : Cela est vrai. On n'est obligé qu'à traiter les gens dans les formes.

(MI, II,5)

28. [R1] ARGAN : Ah ! J'étais bien étonné si l'on ne me parlait pas de la pauvre femme, c'est toujours elle qui fait tout, il faut que tout le monde en parle.

[R2] BÉRALDE : Ah ! J'ai tort, il est vrai, c'est une femme qui a trop d'amitié pour vos enfants; et qui pour l'amitié qu'elle leur porte, voudrait les voir toutes deux bonnes Religieuses.

(MI, III,5)

En tombe le masque [R2] pour le travestissement [R4], de fait, [R2] et [R4] sont contradictoires : en [R2] Sganarelle laisse entendre qu'il n'est pas tout à fait en accord avec la position de Dom Juan, alors qu'en [R4] il bat ouvertement en retraite. De plus la formulation impersonnelle qui prolonge la réplique presque proverbiale de Sganarelle, introduit ON-loc qui vient confirmer la position d'accord factice de Sganarelle. 
106 En, Valère semble se ranger à l'argument d'Harpagon qu'il répète (sans dot) comme un discours rapporté. Son jeu est tel que même le troisième personnage en scène, Elise, n'accède pas à l'interprétation dissensuelle et doute de la sincérité de son amant: «Vous moquez-vous, Valère, de lui parler comme vous faites?». Le dissensus (et par là le comique) vient de l'inadéquation entre l'argument effectivement avancé sans dot et ce qui en est dit une raison tout à fait convaincante : le fait de pouvoir marier sa fille sans dot n'est pas un argument recevable en soi, il ne l'est que pour l'avare Harpagon.

L'exemple montre également un travestissement très rentable puisque non relevé par l'interlocuteur ([R3] cela est vrai); ici, [R2] va dans le sens de [R1] ce qui rend toute l'argumentation absurde. Toinette reprend - en allant plus loin - la pensée de Monsieur Diafoirus.

Enfin en Béralde feint de prendre la défense de l'épouse d'Argan, alors que ON-All sait qu'il joue contre elle dans l'intérêt d'Argan. Les propos qu'il tient pourraient être imputés à Argan lui-même.

Il est à noter que sans contexte, nous n'accèderions pas au dissensus dans ces exemples.

110 Du point de vue de la description, ces exemples posent un certain nombre de problèmes: en effet, doit-on considérer d'emblée que LOC emprunte son discours à l'allocutairepersonange et transformerait par conséquent cet allocutaire en locuteur représenté. Si c'est clairement le cas de, c'est moins évident dans les autres exemples, car en , et respectivement Sganarelle, Toinette et Béralde reformulent - voire donnent voix - aux points de vue de leurs allocutaires, transformant ces derniers en LR.

De plus, comment comprendre que l'accord initial est factice, devons-nous introduire dans la description - au risque de l'alourdir - un locuteur interpréteur qui doublerait la voix du premier Ln? C'est le choix que nous ferons ici, suivant ce que nous avions proposé dans Saez (2017) pour le désaccord masqué, car cela permet d'interpréter une antiphrase par référence dans $M$ aux connaissances de l'œuvre, antiphrase confirmée par la reprise - ou reformulation - des propos de LR. La référence à $\mathrm{M}$ permet de distinguer la présence de LR, et cette présence vient confirmer la position de l'interpréteur. Par conséquent, les propos (pseudo-) rapportés de LR sont interprétés comme une critique du locuteur à l'égard de son allocutaire, et par là, ON-All infère une discordance entre LOC et ALL.

Analyse dans le modèle :

\begin{tabular}{|c|c|c|c|}
\hline & teur & \multicolumn{2}{|c|}{ Récepteur } \\
\hline \multicolumn{4}{|c|}{ Occurrence : ex. [R4] } \\
\hline L1 & $\begin{array}{l}\text { responsable pdv1: c'est fort bien fait à vous, et vous } \\
\text { le prenez comme il faut. }\end{array}$ & \multirow{3}{*}{$\begin{array}{l}\text { ON- } \\
\text { All }\end{array}$} & \multirow{2}{*}{$\begin{array}{l}\mathrm{M}_{\text {\{épistémè\} }}: \mathrm{pdv1} \text { inadéquat } \\
\text { rétablit } \quad \text { c.p. : } \quad \mathrm{pdv} 1= \\
\text { antiphrase }\end{array}$} \\
\hline L1i & responsable pdv2 ss-e : pdv1 injustifié & & \\
\hline LR & $\begin{array}{l}\text { responsable pdv3 : il n'est rien en ce monde que de } \\
\text { se contenter. }\end{array}$ & & $\begin{array}{l}\text { transforme ALL en LR tel } \\
\text { que LR vs } L 1 i\end{array}$ \\
\hline
\end{tabular}




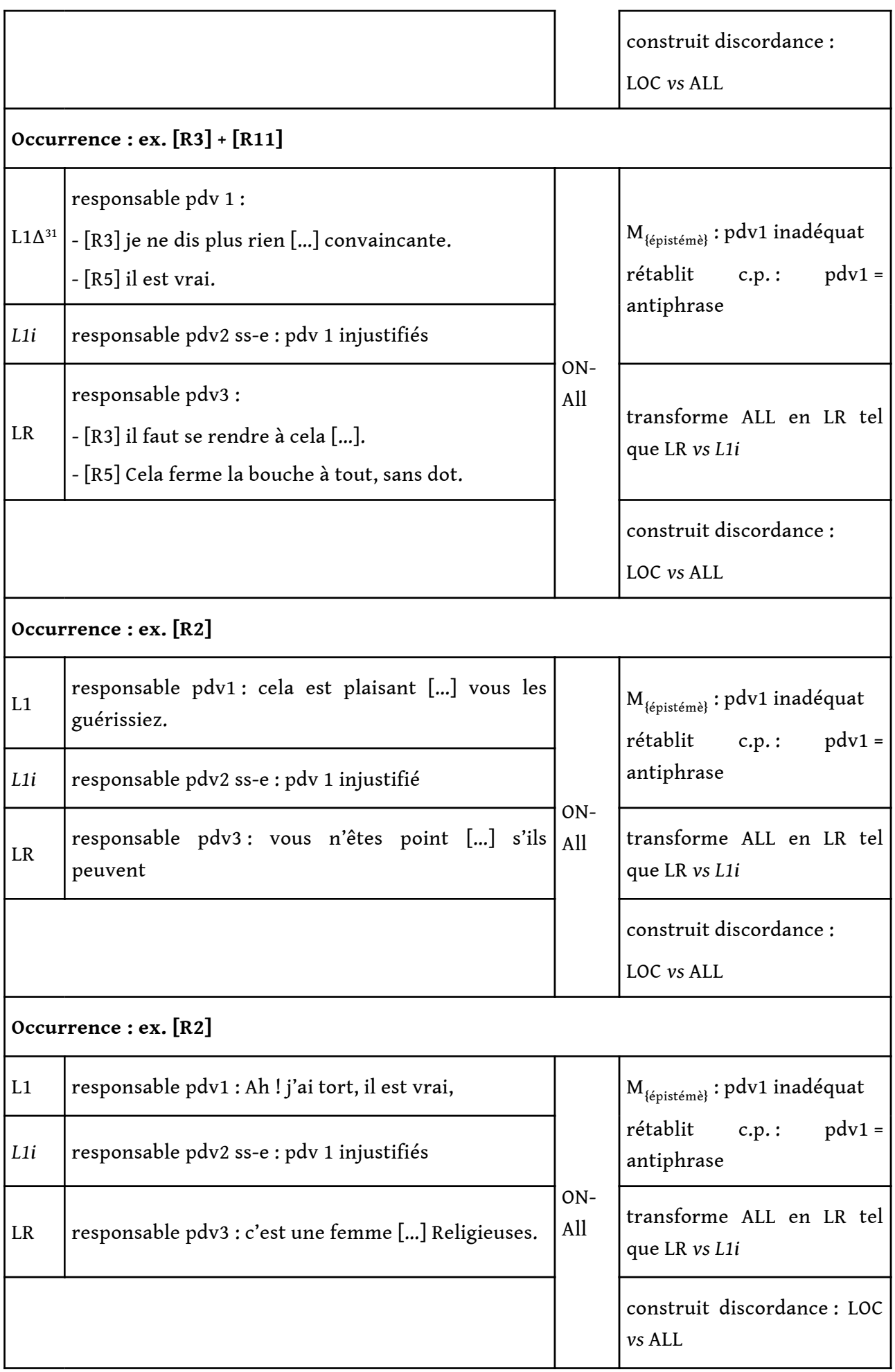

Ici, une sorte de connivence est instaurée entre LOC et le public en raison de la nécessaire interprétation de l'inadéquation entre la situation et le discours de LOC. Ce dernier parie que ON-All comprendra son désaccord car ce qu'il exprime frôle l'absurde (cf. .

On peut donc considérer ON-All comme un co-énonciateur dans la mesure où c'est lui qui restitue l'acceptabilité de l'énoncé : c'est donc l'interprétation discordancielle par ON-All qui assure ici la cohésion polyphonique et donc une partie de la construction du sens. 


\section{Conclusion}

Cette analyse du désaccord travesti a permis de montrer que les phénomènes de polyphonie sont centraux, car ce sont eux qui ouvrent l'interprétation dissensuelle. C'est également ce que nous avions remarqué dans le cadre de notre étude du désaccord masqué.

116 Le tableau ci-dessous reprend nos conclusions ultérieures sur le désaccord masqué en regard de celles du désaccord travesti, soit les différents types de masque ou de travestissement possible pour LOC lorsqu'il souhaite voiler un désaccord et les effets de ces masques selon que le désaccord est suggéré, dit ou montré :

\begin{tabular}{|c|c|c|}
\hline Stratégie discursive & Masques & Travestissement \\
\hline \multirow{3}{*}{$\begin{array}{l}\text { Suggère un pdv } \\
\text { discordant }\end{array}$} & modalité exclamative & $\begin{array}{l}\text { ambiguïté sémantique }+ \\
\text { référentielle } \\
\text { (polyphonie interne et externe) }\end{array}$ \\
\hline & $\begin{array}{l}\text { anaphore associative (polyphonie } \\
\text { externe) }\end{array}$ & connecteur + polyphonie interne \\
\hline & $\begin{array}{l}\text { question rhétorique } \\
\text { (polyphonie interne) }\end{array}$ & $\begin{array}{l}\text { modalité interrogative } \\
\text { (polyphonie interne) }\end{array}$ \\
\hline \multirow{2}{*}{ Dit un pdv discordant } & $\begin{array}{l}\text { présence d'un discours « autre » } \\
\text { (polyphonie externe/ouverte) }\end{array}$ & $\begin{array}{l}\text { didascalies } \\
\text { (polyphonie ouverte) }\end{array}$ \\
\hline & $\begin{array}{l}\text { effet Jekyll et Hyde } \\
\text { (polyphonie (c)ouverte) }\end{array}$ & $\begin{array}{l}\text { aparté } \\
\text { (polyphonie ouverte) }\end{array}$ \\
\hline $\begin{array}{l}\text { Montre un pdv } \\
\text { discordant }\end{array}$ & & $\begin{array}{l}\text { discours rapporté } \\
\text { (polyphonie couverte) }\end{array}$ \\
\hline
\end{tabular}

117 Ce tableau révèle que les marqueurs linguistiques sont presque identiques dans le masque ou le travestissement. Il conviendrait de confronter ces résultats à un corpus de français contemporain - voire de français parlé - afin de mesurer si ces indices langagiers sont propres à un genre ou bien s'ils peuvent constituer des éléments d'identification fiable du processus de désaccord voilé. Cela correspond à notre intuition (entre autres car la langue de Molière nous parait encore très actuelle), mais il conviendrait de la confronter à un corpus.

118 Au cours de ces deux études, nous avons essayé de cerner le rôle de ON-All qui est encore largement à définir. En effet, pour le corpus choisi il nous semble qu'il faille distinguer au moins deux fonctionnements :

29. ON-All interprète à partir d'indice (masques) le désaccord, il serait alors assimilable aux interprétateurs de la ScaPoLine, puisqu'il active des sens disponibles (ici dissensuel) ; il est donc un récepteur actif. Son rôle se situe donc au niveau macro-sémantique ${ }^{32}$. 
30. ON-All construit le désaccord à partir de l'identification d'un travestissement, mais pourrait tout aussi bien ne pas le construire. Ce serait alors également un interprétateur, mais doublé d'un constructeur de sens, il devient co-énonciateur. Son rôle se situe au niveau pragmatique ou - oserions-nous ? - pragma-sémantique dans le sens où il co-construit, en interpréteur des implications praxéologiques, les actions communicatives exercées sur $\mathrm{M}$.

Cette distribution des rôles de ON-All serait à affiner, mais pourrait constituer une base de travail.

Dans cette étude, il resterait également à définir très précisément les rôles des voix auteurs et censure : on pourrait dire rapidement que la voix-auteur est présente dans les didascalies. Mais de quel 'auteur' parler : l'Auteur? une image de l'auteur construite par lui-même? autre chose?

l en va de même pour la censure, elle joue indirectement un rôle : elle peut s'assimiler parfois à ON-All, mais pourquoi ne pas imaginer qu'elle est également présente, notamment en cas d'antiphrase? De fait, la censure serait-elle une instance interprétative ou parlante?

Une hypothèse théorique se dégage également de cette étude : nous avons considéré le désaccord - ou l'interprétation discordancielle - comme gage de la cohérence polyphonique, alors il faudrait peut-être également le considérer comme une macrostructure discursive : un argument allant dans ce sens serait la multiplication des marques pour l'interprétation du désaccord voilé, dont par ailleurs l'ironie peut faire partie.

Nous espérons que cette étude a pu contribuer à la compréhension du phénomène de désaccord voilé, mais elle présente au moins l'avantage de circonscrire un certain nombre de points et d'ouvrir des perspectives de recherches ultérieures.

\section{Corpus}

Les œuvres complètes de Molière dans l'édition de Bibliothèque de La Pléiade 2010 ont été utilisées dans l'étude (comédies et farces), mais pour la présence communication, voici les textes d'où sont issues les occurrences :

Dom Juan (DJ), première représentation le 15 février 1665 ;

Le Misanthrope (M), première représentation le 4 juin 1666 ;

L'Avare (A), première représentation le 9 septembre 1668 ;

Le Tartuffe (T), première représentation dans le texte définitif 1669 ;

Les Femmes savantes (Fs), première représentation le 11 mars 1672;

Le Malade imaginaire (MI), première représentation le 10 février 1673.

BIBLIOGRAPHIE

Anscombre J.-Cl. (2009) « La comédie de la polyphonie et ses personnages », Langue française 2009/4, n 164, pp. 11-31, en ligne. 
Apothéloz D. (1995) « Nominalisation, référents clandestins et anaphores atypiques », TRANEL. Travaux Neuchâtelois de Linguistique, $\mathrm{n}^{\circ} 23$, pp. 143-173, en ligne.

Apothéloz D. \& [Reichler-]Béguelin M.-J. (1995) « Construction de la référence et stratégies de la désignation », TRANEL. Travaux Neuchâtelois de Linguistique, n² 23, pp. 227-271, en ligne.

Authier-Revuz J. (2004) «La représentation du discours autre : un champ multiplement hétérogène ", in Lopez Muños, J. M., Marnette, S. \& Rosier, L. (éds), Le discours rapporté dans tous ses états, Paris : L'Harmattan, pp. 35-53.

Bakhtine M., [1934] (1978) « Du discours romanesque », in Esthétique et théorie du roman, Paris : Gallimard, Tel, pp. 83-233.

Bakhtine M., [1953] (1984) «Les genres du discours », in Esthétique de la création verbale, Paris : Gallimard, pp. 265-308.

Berrendonner A. (1981) Eléments de pragmatique linguistique, Paris : Ed. de Minuit.

- (1990) « Pour une macro-syntaxe », Travaux de linguistique 21, pp. 25-36.

- (2002a) « Les deux syntaxes », Verbum XXIV, pp. 23-35.

- (2002b) « Morpho-syntaxe, pragma-syntaxe, et ambivalences sémantiques », in Leth Andersen, Hanne \& Henning Nølke, éds., Macro-syntaxe et macro-sémantique, Berne, Peter Lang, pp. 23-41.

Boal A. (1996) Théâtre de l'opprimé, La Découverte, coll. « Poche essai », Paris.

Bres J. \& Mellet S. (2009) « Dialogisme et marqueurs grammaticaux », Faits de langue 2009/3, n $\circ 163$, en ligne.

Cappeau P. \& Lay M.-H. (2017) « Pourquoi dire qu'on dit ? Ou négocier le dire », in actes du XXXIe colloque du CerLico, Rennes : Presses Universitaires de Rennes.

Charolles M. (1978) «Introduction aux problèmes de la cohérence des textes [Approche théorique et étude des pratiques pédagogiques] » in Langue française, $n^{\circ} 38$, Charolles M. \& Peytard J. (dir.) Enseignement du récit et cohérence du texte, pp. 7-41.

Dostie G. (2004) Pragmaticalisation et marqueurs discursifs, Bruxelles, De Boeck Supérieur, «Champs linguistiques ».

Ducrot O. (1984) Le dire et le dit, Paris : Éditions de Minuit.

Fløttum K. (2002) «La polyphonie dans une perspective macro-sémantique », in Leth Andersen, Hanne \& Henning Nølke, éds., Macro-syntaxe et macro-sémantique, Berne, Peter Lang, pp. 337-359.

- (2000) « Notes sur la problématique des niveaux de l'analyse polyphonique - de la phrase au texte », Polyphonie II - oktober, http://www.hum.au.dk/romansk/polyfoni

- (2001) «Les liens énonciatifs : tentative d'une nouvelle typologie », Polyphonie III-maj 2001, http://www.hum.au.dk/romansk/polyfoni

Kerbrat-Orecchioni C. (1991) Les interactions verbales, t. 1, Paris : A. Colin.

Lemaréchal A. (1989) Les parties du discours. Sémantique et syntaxe, Paris : P.U.F. (Linguistique nouvelle).

Nølke H. (1994) Linguistique modulaire : de la forme au sens, Louvain : Peeters.

- (2001) « La ScaPoLine 2001, version révisée de la théorie Scandinave de la Polyphonie Linguistique », Polyphonie III-maj, http://www.hum.au.dk/romansk/polyfoni 
Olsen M. \& Nolke H. (2000) « Polyphonie : théorie et terminologie », Polyphonie linguistique et littéraire, pp. 45-171.

Saez F. (2017) «Le désaccord voilé : l'exemple de la comédie de mœurs chez Molière », in actes du XXXIe colloque du CerLiCO, Rennes : Presses Universitaires de Rennes.

\section{NOTES}

1. XXXIe colloque du CerLiCO, Accord, désaccord et non accord, 9-10 juin 2017, Poitiers.

2. Elément pouvant être d'ordre linguistique, périverbal ou contextuel.

3. La construction morphologique par dérivation préfixale même du terme désaccord induit la présence (antérieure ou postérieure) d'un accord exclu.

4. Saez (2017).

5. Au sens de Lemaréchal (1989).

6. Nous présentons les notions utilisées sans les discuter. Le cas échéant, une discussion sera proposée dans l'analyse.

7. Pour l'instant indéterminé, d'où élément.

8. Olsen \& Nølke (2000: 68) parlent de cohésion polyphonique, qui pour les auteurs se situe au niveau textuel. Dans les exemples de désaccord travesti, cette cohésion est (re)construite par ONAll par l'identification du désaccord entre les personnages sur scène.

9. Que nous avons conscience ici de simplifier outrageusement, mais la polyphonie ne fait pas en soi l'objet du présent article.

10. Nølke (2001) distingue trois images de LOC $: l_{0}=$ image de LOC comme locuteur de l'énoncé, $l_{i}=$ image de LOC comme auteur d'une énonciation antérieure ou postérieure à celle de $l_{0}$, et $L=$ image de LOC comme locuteur textuel.

11. Où $n$ correspond au numéro de l'énoncé.

12. Cette distinction rejoint celle de Ducrot (1984) entre le locuteur-en-tant-que-tel et le locuteur-entant-qu'être-du-monde. Olsen \& Nølke (2000: 55) interrogent ces étiquettes préférant celles de locuteur phrastique ou locuteur textuel que nous leur empruntons.

13. Parce que ce n'est pas l'objet de notre propos, nous ne discuterons pas de la pertinence des entités énonciateur et destinataire, et référons aux travaux de Nølke (2001) ou Anscombre (2009) pour quelques pistes de réflexion.

14. Dans la ScaPoLine, respectivement a) responsabilité et b) non-responsabilité.

15. Dans la ScaPoLine, ON-Loc est systématiquement présent : il accorde le posé et le présupposé des pdv.

16. Le public est en effet central dans le genre dramatique ce qui impose de postuler systématiquement la présence de deux ALL simultanés : le personnage à qui s'adresse le locuteur, et le public qui reçoit l'œuvre et participe à différents niveaux.

17. Où $\mathrm{R}=$ réplique. Dans tous les exemples nous soulignons. Pour une analyse complète de cet exemple v. Saez (2017).

18. Cf. Saez (2017) pour une description plus précise de ces indices anaphoriques et leurs rôles dans le dissensus axiologique Sganarelle-Dom Juan.

19. D'où l'effet comique.

20. Cf. Saez (2017).

21. Symbole emprunté à la grammaire générative qui signale que la description est écourtée afin de ne pas alourdir la démonstration, mais ici serait à considérer, entre autres, le conditionnel.

22. Dom Juan est ici transformé en AT, phénomène extrêmement utilisé dans le désaccord masqué (cf. Saez, 2017).

23. Toute prudence gardée vis-à-vis des paraphrases. 
24. La présence du si introduit la présence de cet $\mathrm{AF}$ que tout oppose à l'allocutaire-personnage.

25. Sur dire, v. P. Capeau et M.-H. Lay (2017).

26. Les majuscules sont des auteurs.

27. Cf. aussi Saez (2017) pour un phénomène équivalent dans le désaccord masqué.

28. Dans le sens où ce n'est pas un personnage existant sur scène, mais uniquement un ê.d. créé par LOC, distinct de lui-même.

29. Terme employé en référence à la théorie du théâtre de l'opprimé, telle que définie par Boal (1996).

30. Ce n'est pas l'objet de la description ici, mais le jeu sur la portée du tort de [R2] à [R6], ainsi que les questions rhétoriques présentes en [R6] auraient appelé un commentaire puisque ce sont, selon nous, des phénomènes qui peuvent relever de la polyphonie.

31. Serait également à considérer ici la négation, l'anaphore aspectuelle une raison, et la question rhétorique en [R5].

32. La notion est ici limitée au rétablissement de la cohérence polyphonique, donc à la construction de la cohérence textuelle.

\section{RÉSUMÉS}

Dans la continuité de recherches antérieures, le présent article se propose d'examiner un autre phénomène de désaccord voilé : le désaccord travesti. Il s'agit des cas où un locuteur suggère un point de vue non congruent par rapport à un élément antérieur ou postérieur.

Ce type de désaccord convenable, fréquent dans la comédie de mœurs chez Molière, impose à l'interlocuteur (allocutaire personnage) - et à l'auditeur (allocutaire public) - une opération complexe de décodage, voire de co-énonciation dans le cas du désaccord travesti. Nous avons donc opté pour une approche praxématique qui rend compte à la fois des phénomènes cotextuels (linguistiques et/ou périverbaux) et des interactions verbales, en tentant de mettre en lumière les mécanismes de production et d'interprétation de ce désaccord par l'analyse des indices (linguistiques et/ou périverbaux) qui ouvrent l'interprétation d'un dissensus. Ces indices relèvent essentiellement de phénomènes de dialogisme et de polyphonie, éléments centraux dans notre problématique. Plus généralement nous avons tenté de mettre en lumière la stratégie discursive mise en place par le locuteur et des différents rôles cognitifs des récepteurs.

As a follow up to previous searches, the aim of this article is to examine another phenomenon of veiled disagreement that is to say : the disguised disagreement. It is about the cases where a speaker suggests a point of view that is not congruent with regard to previous (or posterior) elements.

This type of 'suitable disagreement', frequently used in the comedy of morals by Molière, imposes on the interlocutor (the character listening) - and to the audience - a complex operation of decoding, or even of co-statement in the case of disguised disagreement. We thus opted for a praxematic approach which reports at the same time cotextual phenomena (linguistic and/or periverbal) and verbal interactions, by trying to highlight the mechanisms of production and interpretation of this disagreement by the analysis of the markers (linguistic and/or periverbal) that allow for the interpretation of a dissensus. These markers fall essentially within the phenomena of dialogism and polyphony, central elements in the case of disguised disagreement. 
More generally we tried to highlight the discursive strategy organized by the speaker and the various cognitive roles of the receivers.

INDEX

Mots-clés : Désaccord travesti - Polyphonie - Dialogisme - Stratégie discursive - Rôle cognitif Keywords : Disguised disagreement - Polyphony - Dialogism - Discursive strategy - Cognitive role

\section{AUTEUR}

\section{FRÉDÉRIQUE SAEZ}

Académie de Guyane 